\title{
Staphylococcus aureus and Atopic Dermatitis: A Complex and Evolving Relationship
}

\author{
Joan A. Geoghegan, ${ }^{1}$ Alan D. Irvine, ${ }^{2,3,4}$ and Timothy J. Foster ${ }^{1, *}$
}

Staphylococcus aureus is frequently isolated from the skin of atopic dermatitis (AD) patients during flares. The normal microbiota is disrupted and the diversity of the microorganisms on the skin is reduced. Many species that produce inhibitors of $S$. aureus growth decline. Strains from $S$. aureus clonal complex 1 are enriched among AD sufferers whereas the CC30 strains most frequently isolated from nasal carriers in the normal population are much rarer in AD. $S$. aureus expresses several molecules that contribute to the intensity of symptoms, including $\delta$-toxin which stimulates mast cells, $\alpha$-toxin which damages keratinocytes, phenol-soluble modulins which stimulate cytokine release by keratinocytes, protein A which triggers inflammatory responses from keratinocytes, superantigens which trigger B cell expansion and cytokine release, and proinflammatory lipoproteins. Proteases contribute to disruption of the epidermal barrier. S. aureus isolated from AD patients adheres to the deformed corneocytes from $A D$ patients in a clumping factor B-dependent fashion. Novel targeted therapies for $A D$ have recently been introduced to clinical practice with many more in development, including monoclonal antibodies that specifically target cytokines and their receptors, and a bacteriophage lysin that eliminates $S$. aureus from AD skin.

\section{Introduction to $A D$}

$A D$ (syn. atopic eczema) is a chronic inflammatory skin condition that is characterized clinically by periodic flares of dry, red, itchy skin lesions [1-3] and pathogenically by a defective skin barrier, recurrent infections, and both local and systemic type 2 immune responses. The disease is common in children and is less prevalent among adults. There is a high prevalence in developed countries of approximately $20 \%$ in childhood, and an increasing prevalence in developing countries, especially in urbanized areas [4]. Increased 'hygiene' in a general sense could result in a decrease in appropriate immune stimulation that can contribute not only to eczema but also to the allergic diseases asthma and allergic rhinitis and is one hypothesis that may explain some of the prevalence data. Other factors associated with urbanized living, such as exposure to pollution, including diesel fumes, indoor heated living, reduced exposure to endotoxin and parasite infection, enhanced use of washing agents that are deleterious to the skin, and other environmental factors are also likely to contribute to the prevalence of AD [5].

$A D$ is a complex disease and can be triggered in individuals from different genetic backgrounds. Genome-wide scans have identified polymorphisms in several different genes controlling skin barrier integrity that are associated with AD (reviewed in [3]). These include loss-of-function

\section{Trends}

The diversity of the skin microbiome is diminished during an $\mathrm{AD}$ flare, with $S$. aureus assuming hegemony.

Proliferation of $S$. aureus during $A D$ flares is encouraged by reduced competition from the microbiota and favourable growth conditions, including higher $\mathrm{pH}$.

S. aureus expresses superantigens, cytolytic $\alpha$-and $\delta$-toxins, phenol-soluble modulins, protein $A$, and several proteases which have roles in $A D$ pathogenesis.

Clumping factor $\mathrm{B}$ promotes adhesion to deformed corneocytes in $A D$ skin, and this is likely to be an important step in colonization.

New treatments, including a lytic enzyme that is specific for $S$. aureus, are in development. One specific monoclonal antibody inhibitor that targets the receptor for the type 2 cytokines IL-4 and IL- 13 has recently been approved for clinical use, and many others inhibitors targeting type 2 cytokines are in development.

${ }^{1}$ Department of Microbiology, Moyne Institute of Preventive Medicine,

School of Genetics and Microbiology, Trinity College Dublin, Dublin 2, Ireland

${ }^{2}$ Paediatric Dermatology, Our Lady's Children's Hospital Crumlin, Dublin 12, Ireland

${ }^{3}$ National Children's Research Centre, Our Lady's Children's Hospital Crumlin, Dublin 12, Ireland

${ }^{4}$ Clinical Medicine, Trinity College Dublin, Dublin 2, Ireland 


\section{Trends in Microbiology}

mutations in filaggrin, a protein that is important in the development of the stratum corneum the outermost anucleate layer of the epidermis that is the interface between the skin and the environment [6].

Current evidence strongly points to disruption of the integrity of the skin barrier and subsequent immune dysregulation in predisposed individuals, with resultant damage to the skin barrier, as the primary pathological drivers in $\mathrm{AD}$. The skin of an $\mathrm{AD}$ patient during a flare has compromised barrier integrity, which increases the likelihood of irritants penetrating the skin and triggering further inflammation, thus creating a vicious cycle.

The microbiota of the skin is important in maintaining immune homeostasis and preventing the growth of pathogens such as S. aureus [7,8]. During an eczema flare the diversity of the normal microflora is diminished, allowing $S$. aureus to proliferate, in part encouraged by a reduction in bacteria with anti-S. aureus activity. S. aureus elaborates several molecules with potential to cause inflammation and to promote further immune dysregulation. Coagulase-negative staphylococci may reduce the risk of $A D$ through mechanisms that are not completely understood but which include displacement of $S$. aureus by elaboration of antimicrobial substances and immune modulation [9-11].

This review critically evaluates the factors that influence the ability of $S$. aureus to colonize the stratum corneum and the role of $S$. aureus products in the pathogenesis of eczema flares, including superantigens, toxins, proteases, and cell-envelope components. New approaches to treatment are also discussed.

\section{Immune Dysregulation in AD}

Atopic dermatitis stems from a genetic predisposition for disturbance of the epidermal barrier of the skin, for example in individuals with defects in the FLG gene encoding filaggrin (reviewed in [3]). The acute phase can be initiated when an allergen or other irritant penetrates the barrier and is processed by skin antigen-presenting cells (e.g., Langerhans cells) (reviewed in [12] and summarized in Figure 1). Keratinocytes are stimulated to release thymic stromal lymphoprotein (TSLP) and other alarmins such as interleukin-25 (IL-25) and IL-33 which expand skin-resident innate lymphoid cells, further amplifying a Th2 response with naïve $T$ cells diverted towards a Th2 response [13]. A characteristic array of interleukins are produced (IL-3, -4, -5, -13, -31). This triggers an immunoglobulin $\mathrm{E}(\mathrm{lgE})$ response with antibodies which react with both the allergen and self-antigens (reviewed in [12]). Indeed, the level of IgE correlates with autoreactive FC\&RI receptors that recognize the Fc region of IgE. Dendritic cells and mast cells bound to IgE molecules are long-lived, so that cells remain primed to react with allergens for some time.

Induction of IL-4, IL-13, and IL-31 downregulates expression of filaggrin and the cornified cell envelope protein loricrin during keratinocyte differentiation which affects corneocyte function and skin barrier integrity [2,14]. IL-31 is particularly pruritogenic (causing severe itching) [15]. The chronic phase of AD occurs with a shift in T cells towards the Th1 subtype and the production of pro-Th1 cytokines such as interferon- $\gamma$, IL-1, and IL-12, along with IL-22 [16]. It can last for years or even a lifetime. The skin is inflamed and itchy, it increases in thickness (lichenification), and is further damaged due to scratching $[1,2]$.

\section{Differences between Healthy and AD Skin}

Healthy skin is desiccated, it has a low pH at the surface, the upper layer is constantly shed removing attached bacteria, and host antimicrobial defensin peptides are present. The skin is a
*Correspondence: tfoster@tcd.ie (J.A. Geoghegan Correspondence:). 


\section{Trends in Microbiology}

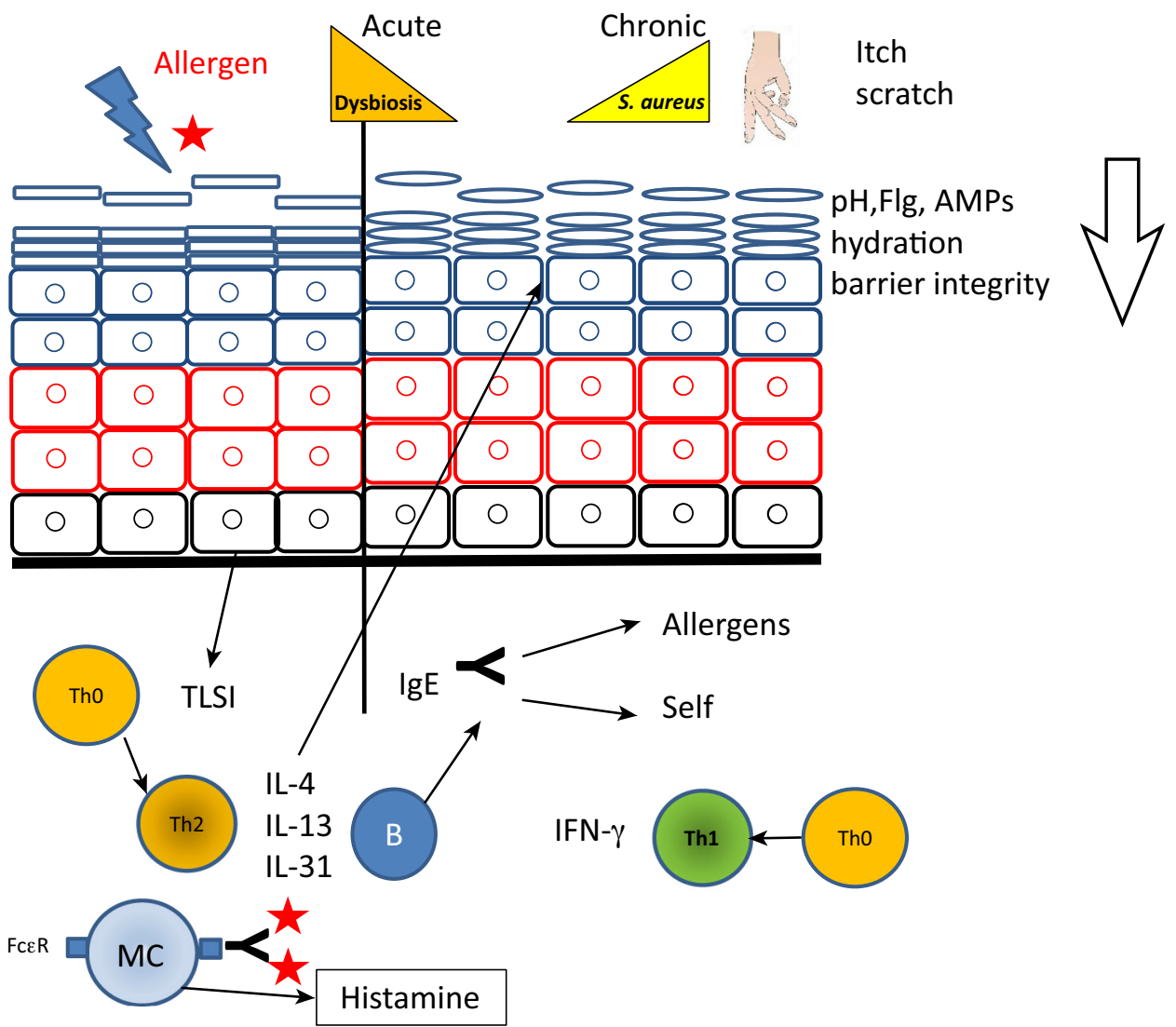

Trends in Microbiology

Figure 1. Changes to Skin in Atopic Dermatitis. Summary of changes that occur in skin following development of atopic dermatitis. Initially, the skin barrier is breeched and an allergen penetrates. This triggers thymic stromal lymphoprotein (TLSP) expression which stimulates Th0 cells to develop into Th2 cells - which, in turn, trigger B cells to produce lgE that reacts with allergens and self-antigens. The Th2 cytokines contribute to symptoms by stimulating pruritis and interfering with corneocyte development and barrier integrity. The normal microbiome is altered (dysbiosis), allowing $S$. aureus to proliferate. As the disease moves into a chronic state, Th1 cells develop, releasing proinflammatory cytokines such as IFN- $\gamma$.

hostile environment for many bacteria and provides a strong barrier to harmful substances and irritants (reviewed in [17] and summarized in Figure 1).

The $\mathrm{pH}$ of skin is crucial for maintaining the epidermal barrier function and controlling desquamation, and it is an important defence against pathogens. The $\mathrm{pH}$ of healthy skin ranges from 4 to 6 and is lowest at the surface. The low pH is maintained by fatty acids that occur in sweat and sebum, by products of phospholipid hydrolysis, and by sodium-proton ion exchangers of lamellar bodies that form during keratinocyte differentiation [18]. The filaggrin breakdown products urocanic acid (UCA) and pyrolidone carboxylic acid (PCA) help to maintain the acidic $\mathrm{pH}$ and contribute to the natural moisturizing factors (NMFs) that control skin hydration [19].

In $\mathrm{AD}$, the $\mathrm{pH}$ of skin shifts toward alkalinity [17]. Several factors contribute to this shift, such as decreased sweat secretion and decreased levels of free fatty acids. Mutations in the FLG gene result in reduced levels of UCA and PCA [20]. The Th2 cytokines IL-4 and IL-13 act synergistically to reduce FLG expression so that levels of UCA and PCA are reduced in AD lesions even in 


\section{Trends in Microbiology}

individuals who are wild type for FLG [20,21]. The antimicrobial defensin peptides LL-37 (cathelicidin), $\beta$-defensins, and dermicidin are present at reduced levels in AD skin (reviewed in [22]). Expression of the human $\beta$-defensin genes HBD-2 and -3 is inhibited by the Th2 cytokines IL-4 and IL-13. These changes likely contribute to dysbiosis of the microbiota and proliferation of $S$. aureus.

\section{Association of $S$. aureus with $A D$}

As discussed, many studies have shown that $S$. aureus is prevalent on the inflamed skin of $A D$ patients [23-27]. The reported rates of carriage can vary from 30 to $100 \%$, depending on the type of patient, the sample size, the method used to take samples, and the method used to detect bacteria (culturing, detecting bacterial products, or using PCR and 16S rRNA sequencing).

A recent meta-analysis of 95 observational studies of culture-based methods showed (i) that the prevalence of $S$. aureus carriage by AD patients was $70 \%$ on lesional skin compared to $39 \%$ on nonlesional skin or healthy control skin [27], and (ii) that the prevalence rate increased with disease severity [27]. Examples of quantitative prevalence studies are described in [23,25].

Using RT-PCR to estimate the burden of $S$. aureus by amplifying a chromosomal gene from DNA extracted from a standard volume of lavage fluid, the density of $S$. aureus on both lesional and nonlesional skin correlated with disease severity [26], confirming results from earlier culture-based studies [23,24].

The anterior nares provides the main habitat for commensal $S$. aureus, which persistently colonizes $20-30 \%$ of the population [28-30]. Several studies of carriage strains from different geographic locations have defined the population structure based on multilocus sequence typing (MLST) clonal complexes and have shown that strains from CC30 occur most frequently in normal populations [31-34]. In contrast, several studies of $S$. aureus strains isolated from the inflamed skin of children and adults with AD have shown that CC30 isolates are underrepresented whilst strains from $\mathrm{CC} 1$ are over-represented compared to healthy controls [34-36]. The features of CC1 strains that enable them to proliferate in AD are not known, although it appears that $\mathrm{CC} 1$ strains are more prevalent on the lesional skin of patients carrying FLG mutations compared to patients without FLG mutations [37]. A higher AD severity was seen in patients colonized with CC1 strains [36].

\section{The Inhibitory and Immumodulatory Activity of the Skin Microbiome.}

\section{Changes to the Microbiome in AD}

Intriguing new insights into the mechanisms behind immune tolerance towards skin commensals came from studying Staphylococcus epidermidis colonization of neonatal mouse skin [38]. Activated $\mathrm{CD} 4^{+} \mathrm{T}$-regulatory cells migrated to the skin during a developmental window which generated tolerance towards the commensal [38]. In adult mice, colonization of the skin with $S$. epidermidis induced IL-17A ${ }^{+} \mathrm{CD} 8^{+} \mathrm{T}$ cells to migrate to the skin, which provided enhanced innate immunity to a pathogen [39]. This indicates that dendritic cells resident in skin can sense changes to the skin microbiota and provide protection against potential pathogens.

Analysis of the skin microbiota by deep shotgun metagenomic sequencing and sequencing 165 rRNA genes has made a major impact on understanding the changes in bacterial composition that occur during an eczema flare. The original studies did not measure bacterial density per se but quantified the relative proportions of different groups of bacteria [40]. 


\section{Trends in Microbiology}

Samples were taken (i) from AD patients who were not displaying any clinical symptoms in order to provide the microbiome baseline, (ii) during eczema flares from patients who were either subjected to conventional treatment or who were untreated, and finally (iii) during the post-flare recovery phase. Microbiome diversity decreased in inflamed atopic skin with reductions in the genera Streptococcus, Corynebacterium, Propionibacterium and the phylum Proteobacteria in favour of members of the genus Staphylococcus in general and S. aureus in particular. Shotgun metagenomic sequencing of samples taken from skin showed that $A D$ patients are usually colonized with a single strain of $S$. aureus during a severe flare [41]. The microbiome composition reverted to normal during treatment and recovery [40]. Thus, successful treatment allows reconstitution of normal microbial diversity, presumably by removing staphylococci (and other bacteria) and allowing the skin to be repopulated with normal microflora, or restores the skin to functionality, allowing the normal microflora to become re-established.

One of the functions of the normal skin microbiota appears to be to suppress the growth of $S$. aureus. Several different mechanisms of interference have been identified. The coagulasenegative staphylococci (CoNS) S. epidermidis, S. hominis and S. lugdunensis can elaborate peptide antibiotics (lantibiotics) that are bactericidal towards S. aureus [10,42]. Furthermore an S. hominis lantibiotic acts synergistically with host antimicrobial peptides [10]. S. epidermidis releases phenol-soluble modulins with specific inhibitory activity towards skin pathogens, including S. aureus, and which act cooperatively with host antimicrobial peptides [11]. CoNS with anti-S. aureus activity are common in normal skin but occur less frequently in AD skin colonized by $S$. aureus. Similarly, the Gram-negative Roseomonas mucosa isolated from healthy skin elaborates a bactericidal substance, most likely a lipid, whereas strains of $R$. mucosa that are (rarely) found on AD skin do not [43]. In the case of S. hominis and R. mucosa, coinoculation with $S$. aureus onto mouse skin reduced the pathogen burden and signs of $A D$ $[10,43]$. Corynebacterium striatum expresses an as yet unidentified factor that interfers with Agr-mediated control of virulence, maintaining expression of toxins such as $\alpha$-haemolysin [44]. Propionibacterium acnes ferments glycerol present in sebum and forms short-chain fatty acids that reduce the $\mathrm{pH}$ [45]. This inhibits the growth of $S$. aureus and could reduce expression of surface protein adhesins and protein A most likely by activating the mild acid stimulon [46].

Carriage of $S$. aureus in the nares is influenced by the normal flora. The presence in the nares of S. epidermidis expressing the Esp protease, and S. lugdunensis that synthesizes the cyclic peptide antibiotic lugdunin, is associated with individuals who are noncarriers [30,42,47]. It is possible that these species could contribute to reducing $S$. aureus on healthy skin. It is thus evident that the normal microbiota contributes, along with host defences and the low $\mathrm{pH}$, to reduce growth of $S$. aureus on healthy skin. The data summarised above strongly suggest that the normal flora suppresses growth of $S$. aureus and that dysbiosis of the microbiota contributes to the pathogenesis of $A D$ [48].

\section{Colonization of AD Skin by S. aureus}

Despite the inhibitory role of the normal skin microbiota in trying to prevent growth of $S$. aureus, S. aureus colonizes AD skin very effectively. S. aureus adheres to skin biopsies taken from the nonlesional skin of patients with $A D$ more efficiently than to skin biopsies taken from patients with the another common inflammatory skin disease (psoriasis) or healthy volunteers [49]. The bacteria primarily adhere to the stratum corneum, where dead flattened cornified keratinocytes known as corneocytes are located. The reason for the preferential adherence of $S$. aureus to $A D$ skin is not understood but changes to the composition of the stratum corneum and corneocyte morphology are likely to contribute. In contrast to healthy skin, fibronectin may be present in the stratum corneum of AD skin and thereby accessible to $S$. 


\section{Trends in Microbiology}

aureus as a ligand [49]. The fibronectin-binding proteins (FnBP) A and B expressed on the surface of $S$. aureus promote bacterial adherence to fibronectin [50] and promote strong adhesion to AD skin biopsy specimens compared to healthy ones [49]. Other known ligands for $S$. aureus are exposed in the stratum corneum. For example, the $S$. aureus cell-wallanchored (CWA) protein ClfB binds to the cornified envelope proteins loricrin and cytokeratin 10 [51] and has recently been shown to promote bacterial adhesion to corneocytes taken from AD patients [34]. The CWA protein IsdA provides resistance to antimicrobial lipids, a property which may contribute to colonisation of $A D$ skin, as well as promoting adhesion to corneocytes [52,53]. Several other surface-located CWA proteins promote adhesion to nasal squamous epithelial cells [54]. Given the similarities between nasal squamous cells and skin corneocytes it is possible that these adhesins could also recognise ligands exposed on corneocytes of AD patients and contribute to colonization. However, corneocyte morphology is grossly altered in AD patients with low NMF [55]. Instead of having a flat surface, villus-like projections protrude from the upper surface of the corneocytes (Figure 2). The tips of the villus-like projections are coated with corneodesmosin, a protein involved in connecting corneocytes and usually confined to the cell-cell junctions in the stratum corneum [55]. The unusual distribution of corneodesmosin in AD corneocytes, and the structural changes accompanying low NMF, suggest that additional ligands may be accessible in the unique niche represented by the stratum corneum of $A D$ skin.

\section{S. aureus Molecules/Products and Components That Contribute to Pathogenesis}

S. aureus expresses a plethora of secreted and wall-anchored virulence factors that have proven roles in the pathogenesis of both superficial and invasive infections $[50,56,57]$. Several have been implicated in the pathogenesis of AD by studying effects on keratinocytes and immune cells both in vitro and from human skin biopsy samples, and in murine allergy models, evidence of which is supported by detection of the factors in AD skin (Table 1). Furthermore, $S$. aureus incorporates short-chain unbranched fatty acids into its cytoplasmic membrane when growing in vivo [58]. This increases membrane fluidity, and could influence the expression of virulence factors [59] and tolerance to host innate immunity, such as resistance to oxidative
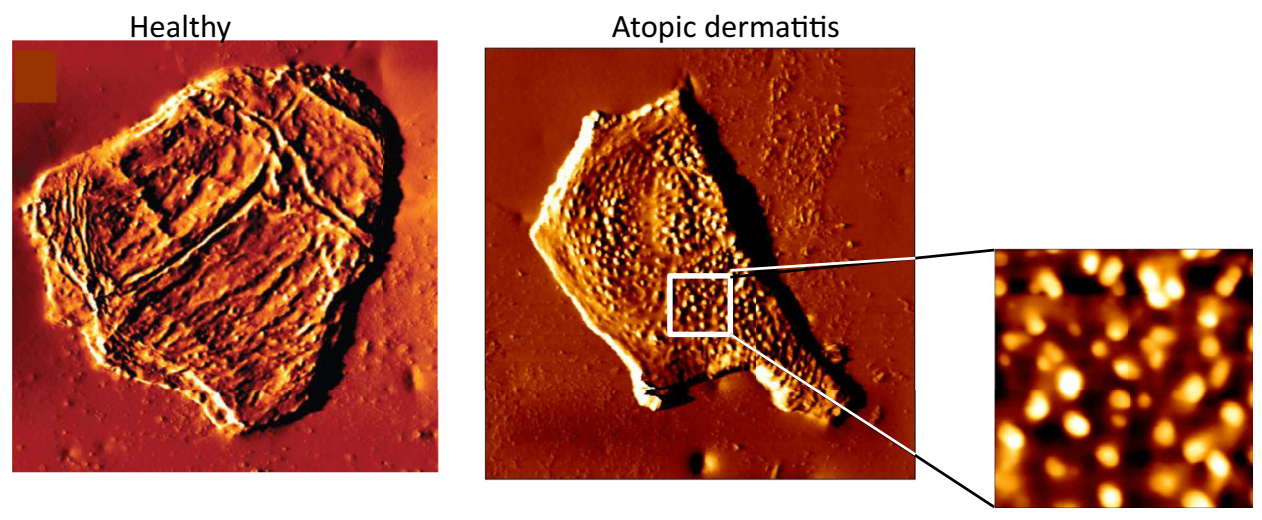

Trends in Microbiology

Figure 2. Corneocyte Morphology. The surface topology of corneocytes from the skin of a healthy volunteer, or from inflamed skin of an AD patient, attached to tape strips used to lift the cells from the skin surface. Contact mode imaging using atomic force microscopy with an unmodified silicon nitride tip was used to scan the surface of the corneocytes. The small panel shows the surface of the AD patient's corneocyte magnified. These images are reproduced with permission from the Royal Society for Chemistry [98] and the American Society for Microbiology [34]. 


\section{Trends in Microbiology}

Table 1. Staphylococcus aureus Proteins That Contribute to Atopic Dermatitis

\begin{tabular}{|c|c|c|}
\hline S. aureus proteins & Possible role in $A D$ & Refs \\
\hline Clumping factor $\mathrm{B}$ & Adhesion to corneocytes in stratum corneum via loricrin or other ligands & [34] \\
\hline $\begin{array}{l}\text { Fibronectin-binding } \\
\text { proteins }\end{array}$ & $\begin{array}{l}\text { Adhesion to fibronectin that is present at high levels in the upper strata } \\
\text { of epidermis and stratum corneum of } A D \text { skin }\end{array}$ & [49] \\
\hline Protein A & Proinflammatory. Binds to TNFR-1 on keratinocytes & {$[78,79]$} \\
\hline Lipoproteins & Proinflammatory. Activate TLR-2 on keratinocytes & {$[60,81]$} \\
\hline$\alpha$-Toxin & Membrane damage/lysis of keratinocytes & [72-74] \\
\hline$\delta$-Toxin & Mast cell degranulation. Synergy with IgE. Allergic skin inflammation & [68] \\
\hline $\begin{array}{l}\text { Phenol-soluble } \\
\text { modulins }\end{array}$ & $\begin{array}{l}\text { Trigger proinflammatory responses associated with } A D \text { in keratinocytes } \\
\text { at sublytic concentrations }\end{array}$ & [69-71] \\
\hline $\begin{array}{l}\text { Enterotoxins and } \\
\text { TSST-1 }\end{array}$ & $\begin{array}{l}\text { Excessive } T \text { cell cytokine production and toxicity. Allergens. Enterotoxins } \\
\text { might trigger mast cell degranulation directly }\end{array}$ & {$[56,64]$} \\
\hline $\begin{array}{l}\text { Staphopain } \\
\text { Aureolysin }\end{array}$ & Inactivation of antimicrobial peptides & {$[84,85]$} \\
\hline V8 serine protease & Epidermal barrier dysfunction in hairless mice & [87] \\
\hline $\begin{array}{l}\text { Serine protease-like } \\
\text { proteins }\end{array}$ & $\begin{array}{l}\text { Potent allergens in idiopathic asthma following S. aureus colonization. } \\
\text { Similar role in AD? }\end{array}$ & [89] \\
\hline
\end{tabular}

stress mediated by staphyloxanthin [58]. Incorporation of skin fatty acids into lipoproteins increases their proinflammatory properties [60].

\section{Superantigens}

All S. aureus strains express superantigens (SAgs) such as toxic shock syndrome toxin-1 (TSST-1) and the staphylococcal enterotoxin serotypes SEA, SEB, SEC, SED, SEE, or SEG [56]. Superantigens bind to major histocompatibility class II (MHCII) molecules on the surface of antigen-presenting cells and T cell receptors on T cells, allowing cells to interact without the constraint of an antigenic peptide presented by $\mathrm{MHCll}$ molecules (Figure 3). This results in excessive production of $\mathrm{T}$ cell cytokines which causes toxicity. In the case of SEB, and most likely other SAgs, the MHCll-T cell receptor complex is stabilized by the SAg forming a quadripartite complex by binding CD28 on the T cell, a finding which helps explain the toxin's extremely high potency [61].

SAgs are also allergens and generate an IgE response. Evidence from clinical studies for the association of SAgs with $A D$ is summarized in Figure 3. Compelling experimental evidence came from studies with SCID mice primed with peripheral blood mononuclear cells from AD patients where topical application of SEB triggered an AD-like response [62].

What has been overlooked as investigators focussed on the superantigenicity of enterotoxins is evidence that they can also interact directly with mast cells. If ingested, enterotoxins cause emesis [63]. The house musk shrew is the only rodent known to be susceptible to ingestion of enterotoxins. In this animal, orally administered SEA passed across the lumen and bound to submucosal FceRI-positive mast cells via an unknown receptor [64]. This induced degranulation and release of serotonin which is recognized by the enteric nerve. Depolarization of this nerve activates afferent vagal nerves which then stimulate the vomiting centre in the brain and the vomiting reflex. 


\section{Trends in Microbiology}

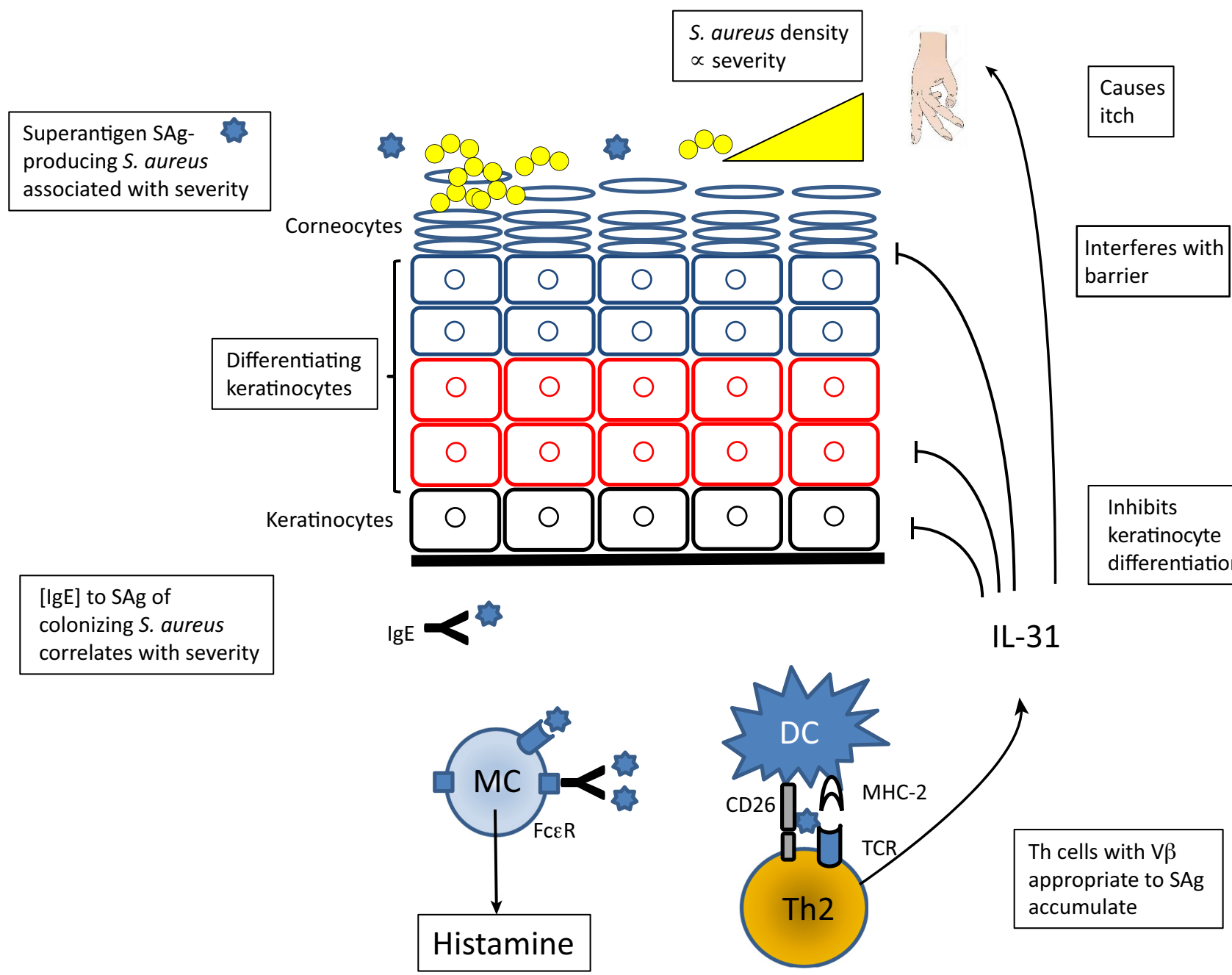

Trends in Microbiology

Figure 3. Role of Superantigens in Atopic Dermatitis (AD). The structure of skin and the effects of superantigen (SAg)-expressing Staphylococcus aureus. The yellow circles represent $S$. aureus colonizing the corneocytes of the stratum corneum. Bacteria synthesize superantigens (SAgs) such as SEA, SEB, SEC, and TSST-1 (blue star). IgE antibodies are raised to the SAgs via normal antigen presentation to Th2 cells and selection and differentiation of B lymphocytes (not shown). The allergen $\mathrm{SAg}$ can then trigger mast cell degranulation via lgE and Fc\&R. Enterotoxins might also trigger mast cell degranulation directly via an unknown receptor. The text boxes summarize the known association between SAgs and the development of AD. Children colonized with bacteria expressing these SAgs had higher disease severity than those colonized with strains lacking these toxins [99]. Disease severity correlated with the density of SAg-expressing bacteria. AD patients produce IgE to the SAg expressed by the colonizing strain, indicating that SAgs are also allergens [100]. Mast cells and basophils from sensitized patients release histamines in response to the SAg expressed by the colonizing strain. The cells are primed with SAg-specific IgE [56]. The level of IgE recognizing the SAg expressed by the colonizing strain correlated with disease severity [101]. SEB stimulates AD-like symptoms when added to the skin of normal individuals and uninvolved skin of AD sufferers. The SAg induced selective accumulation of T cells expressing $V \beta$ repertoires appropriate to the toxin [102]. SAgs induced Th2 cells to release IL-31, which inhibits keratinocyte differentiation, downregulates filaggrin expression - which contributes to a reduction in skin barrier function, and stimulates itchiness [14].

In Cynomolgus macaques, ingestion of enterotoxins also triggers an emetic response. The application of very low concentrations of SEB to the skin of macacques triggered immediatetype hypersensitivity associated with degranulation of skin mast cells $[65,66]$. The monkeys had no detectable antibodies to SEB. The skin reaction was inhibited by histamine $\mathrm{H} 2$ receptor agonists. These observations raise the possibility that enterotoxins might activate skin mast cells by a mechanism similar to those in the gastric mucosa and could thus contribute to AD symptoms by two distinct mechanisms. 


\section{Trends in Microbiology}

$\delta$-Toxin and Phenol-Soluble Modulins

S. aureus can express several short amphipathic peptides called phenol-soluble modulins (PSMs), a group of molecules comprising the PSM $\alpha$ and $\beta$ families along with $\delta$-toxin that is encoded within the regulatory RNAlll of the cell-density-dependent global accessory generegulatory system (Agr) [67].

$\delta$-Toxin can specifically cause degranulation without lysis of murine mast cells derived from fetal skin or bone marrow [68]. In contrast, PSM $\alpha 2$ and PSM $\alpha 3$ caused mast cell death. $\delta$-Toxin acted synergistically with murine $\mathrm{lgE}$ in the absence of bound antigen. Furthermore, in a murine model of allergic skin disease, $S$. aureus triggered inflammation in a $\delta$-toxin-dependent fashion. A mutant lacking the ability to express $\delta$-toxin was almost completely defective, while activity was restored with a complementing $\delta$-toxin-expressing plasmid. These experiments did not directly address the expression of PSMs in vivo but nevertheless demonstrated an important role for $\delta$-toxin. Importantly, $\delta$-toxin was detected in the lesional skin of AD patients [68].

Alpha-type PSMs activate proinflammatory cytokine expression by human keratinocytes and in murine skin colonization models which cause skin damage and inflammation associated with AD [69-71]. Importantly, these effects were only seen following epicutaneous application and not following subcutaneous injection, which triggered a different response.

$\alpha-$ Toxin

$\alpha$-Toxin is a potent pore-forming cytolysin, and is a proven virulence factor in several animal models of infection [57]. The high-affinity receptor for $\alpha$-toxin on mammalian cells is the matrix metalloprotease ADAM-10. Toxin monomers bind to ADAM-10 and activate the nascent ADAM-10 protease which then cleaves E-cadherin in epithelial cell tight-junctions. As the concentration of toxin builds up it oligomerizes in the membrane in a sphingomyelin-dependent fashion to form pores which cause lysis and cell death. Immunohistochemical analysis has shown $\alpha$-toxin in the dermis of AD patients colonized with S. aureus [72]. Keratinocytes in AD skin biopsy samples are more susceptible to $\alpha$-toxin-promoted killing than are those from healthy individuals [73].

During keratinocyte differentiation filaggrin expression is induced [21]. This is associated with secretion of sphingomyelinase that reduces keratinocyte susceptibility to $\alpha$-toxin by decreasing the level of the lipid needed for lysis [74]. In AD, high levels of Th2 cytokines reduce filaggrin and sphingomyelinase expression, making differentiating keratinocytes more susceptible to $\alpha$-toxin. This could contribute to loss of barrier function and to increased penetration of irritants and allergens. It can be concluded that $\alpha$-toxin is likely to play an important role in disrupting the skin barrier in the skin of eczema patients that is colonized by $S$. aureus.

Protein A

Protein $A$ is a multifunctional CWA protein that is both covalently anchored to peptidoglycan by sortase $A$ and released into the extracellular milieu $[50,75,76]$. Protein $A$ has five domains which recognize several different ligands, including tumour necrosis factor receptor-1 (TNFR-1). Binding to, and activating, TNFR-1 induces a proinflammatory response [77]. TNFR-1 is highly expressed by human keratinocytes, and protein $A$ is readily detectable in skin biopsy samples from patients with $A D[78,79]$. Therefore, it is very likely that secreted protein $A$ contributes to inflammation in patients who are heavily colonized by S. aureus. 


\section{Trends in Microbiology}

Lipoprotein and Lipoteichoic Acid

Lipoteichoic acid (LTA) obtained from commercial sources is usually contaminated with lipoproteins which are now known to be potent TLR-2 agonists [80,81]. Therefore, any publications purporting to show proinflammatory effects of LTA should be treated with caution. LTA has been detected in lavage fluid from AD patients, and its concentration correlates with disease severity [82]. However, this could just be a manifestation of the bacterial burden and the corresponding high expression of protein toxins or released lipoproteins. Purified synthetic diacylated lipopeptide induced TSLP expression by primary human keratinocytes in a TLR-2/ TLR-6 dependent manner so it is likely that liproproteins can contribute to the triggering and maintenance of the Th2 allergic response in AD [83], a process that is enhanced by incorporation of unbranched fatty acids acquired in the skin into the lipoproteins [60].

\section{Proteases}

The cysteine protease staphopain and the metalloprotease aureolysin cleave the antimicrobial peptide LL-37 $[84,85]$. Staphopain was detected in AD biopsy samples in close association with $S$. aureus cells which were growing in a biofilm on the surface of the stratum corneum [84]. Staphopain and other proteases might act in concert to protect $S$. aureus from antimicrobial peptides (AMPs) in AD skin.

S. aureus proteases promoted bacterial penetration of the epidermal barrier in a human skin equivalent model [86], and the V8 serine protease caused epidermal barrier dysfunction when applied to the skin of hairless mice [87]. S. aureus also stimulated human keratinocytes to increase expression of endogenous proteases, which also contributed to barrier disruption [88]. Thus, both bacterial and host proteases perturb the integrity of the skin barrier.

\section{Bacterial Allergens}

Patients suffering from idiopathic intrinsic asthma have a high rate of colonization by $S$. aureus in the upper airway. Immunoblotting analysis of the exoproteome of $S$. aureus with serum from patients revealed that the staphylococcal serine protease-like (Spl) proteins reacted strongly with IgG4, an IgG subclass that is a surrogate marker for a Th2 driven immune response [89]. IgE specific for Spls was detected in serum from the majority of sufferers. Furthermore, recombinant SpID administered to mice intratracheally elicited allergic lung inflammation with antigen-specific lgE and elevated Th2 cytokines [89]. It would be very interesting to perform a similar study with the serum from AD patients. This would determine if Spls are prominent allergens in $A D$ as well as asthma.

\section{Treatment of AD}

The objective of treatment is to reduce inflammation and itching and to restore the appearance and function of skin to normal. Conventional treatments that have not advanced much over the past 20 years involve emollients (medical moisturizers) and topical anti-inflammatory corticosteroids and calcineurin inhibitors [90]. There are limitations to steroid usage because of concerns over long-term usage causing skin atrophy and systemic side-effects, and limited patient tolerance for extensive and prolonged topical application regimens. For more severe cases, nonspecific immunosuppressants such as cyclosporine, azathioprine, or mycophenylate have been used with varying degrees of success [91].

A plethora of pathogenesis-based treatments have been, or are being, developed that promise to provide more specific target-based treatments and also to advance understanding of the pathophysiology of the disease (reviewed in [92]). These include monoclonal antibodies that neutralize IgE or block specific interleukins or their receptors, and small-molecule inhibitors of 


\section{Trends in Microbiology}

enzymes in inflammatory signalling pathways such as the JAK STAT pathway. The most advanced is the IL-4 receptor blocker dupilumab which has undergone a successful phase III trial and is now approved for clinical use in the USA. This molecule inhibits the function of IL-4 and IL-13, important mediators of the Th2 pathway.

Bleach baths have been widely used in the treatment of AD in the USA and in other countries for decades but the evidence base is limited to a single clinical trial [93]. Topical and systemic antibiotics have been employed in attempts to reduce the burden of $S$. aureus and its toxic and proinflammatory products in AD skin. However, until recently, no properly conducted clinical trials have been performed on the efficacy of antibiotics in treating AD. In a randomized clinical trial conducted in the UK, topical fusidic acid was compared to systemic flucloxacillin or erythromycin in children with clinically infected eczema and showed no benefit over and above the effects of standard-of-care emollients and corticosteroids [94]. This result does not support routine administration of antibiotics in treating $A D$ in the absence of overt clinical infection.

A bacteriophage lysin called Staphefekt is currently undergoing a phase III trial as a topical agent for treating eczema in adults. The advantages of the lysin compared to fusidic acid are (i) it is rapidly bactericidal, (ii) it is specific for $S$. aureus, and (iii) resistance is unlikely to occur during treatment [95-97]. This trial should determine if eliminating $S$. aureus by this more targeted approach, compared to topical antibiotics, has a beneficial effect in alleviating symptoms and promoting recovery.

\section{Concluding Remarks}

S. aureus is frequently isolated from the inflamed skin of $A D$ patients. Its proliferation is accompanied by a reduction in the diversity of bacteria that normally comprise the skin microbiota, resulting in a dearth of organisms expressing substances that inhibit the pathogen. Inflammation is exacerbated by $S$. aureus due to the elaboration of toxins and lipoproteins that affect keratinocytes and skin immune cells (Table 1).

The changes to AD skin that allow $S$. aureus to proliferate are likely to include elevated $\mathrm{pH}$, increased surface exposure of tissue fluid and blood as binding targets, a reduction in host antimicrobial peptides, and an altered microbiota. Changes in the morphology and surface composition of corneocytes might provide a greater abundance of ligands to which $S$. aureus can adhere $[34,55]$.

Several studies have shown that strains most commonly isolated from the inflamed skin of AD patients' skin are from CC1, whereas strains from CC30 which are most frequently isolated from the nares of healthy carriers are under-represented in $A D$ [34-36]. A detailed investigation into the factors that contribute to the success of CC1 strains in AD lesions should be carried out, for example, by comparing the genome sequences of a panel of CC1 and CC30 strains combined with proteomic and transcriptomic analysis.

CC1 strains from AD patients adhere strongly to the corneocyte ligand loricrin. Enhanced adhesion to corneocytes could thus be a contributing factor to the success of CC1 strains. Future research should focus on the involvement of other adhesins and the possibility that the deformed corneocytes from AD skin express ligands to which CC1 strains are better equipped to adhere. Also nutrients in AD skin might differ from the nasal habitat. It is possible that $C C 1$ strains can grow better there than CC30 strains.

\section{Outstanding Questions}

What are the conditions that trigger changes to the skin microbiota at the beginning of an $\mathrm{AD}$ flare?

What are the full repertoire of factors elaborated by competing microbiota that inhibit the growth of $S$. aureus in normal skin?

What other adhesins are important in colonization of $\mathrm{AD}$ corneocytes?

Which S. aureus proteases are responsible for disruption of the epidermal barrier?

Can blocking adhesion of $S$. aureus to corneocytes contribute to a reduction in the $S$. aureus burden during $A D$ flares?

What are the features of $\mathrm{CC} 1$ strains that allow them to proliferate so well compared to the CC30 strains that predominate in healthy nasal carriers?

What are the major allergens elaborated by $S$. aureus?

Can treatment of $A D$ be improved by eliminating $S$. aureus?

Is there a role for vaccination against $S$. aureus in atopic individuals? 
Trends in Microbiology

The wide body of evidence reviewed above suggests that elimination of $S$. aureus through targeted therapies, or modification of the commensal microbiota, presents a promising area of exploration in either the prevention or modification of AD. Indeed, a very recent clinical trial has provided evidence that microbiome manipulation deserves further exploration [10].

In conclusion, it is now clear that proliferation of $S$. aureus contributes significantly to the pathogenesis of $A D$ flares by elaboration of several proinflammatory and immunosuppressive factors. Adhesion by $S$. aureus to deformed corneocytes in AD skin is an important first step. Further knowledge about the role of $S$. aureus in AD will lead to better treatment options (see Outstanding Questions).

\section{Resources}

'https://clinicaltrials.gov/ct2/show/NCT02840955

\section{References}

1. Bieber, T. (2008) Atopic dermatitis. N. Engl. J. Med. 358, 14831494

2. Weidinger, S. and Novak, N. (2016) Atopic dermatitis. Lancet 387, 1109-1122

3. Boguniewicz, M. and Leung, D.Y. (2011) Atopic dermatitis: a disease of altered skin barrier and immune dysregulation. Immunol. Rev. 242, 233-246

4. Flohr, C. and Mann, J. (2014) New approaches to the prevention of childhood atopic dermatitis. Allergy 69, 56-61

5. Langan, S.M. and Irvine, A.D. (2013) Childhood eczema and the importance of the physical environment. J. Invest. Dermatol. 133, 1706-1709

6. Irvine, A.D. et al. (2011) Filaggrin mutations associated with skin and allergic diseases. N. Engl. J. Med. 365, 1315-1327

7. Powers, C.E. et al. (2015) Microbiome and pediatric atopic dermatitis. J. Dermatol. 42, 1137-1142

8. Williams, M.R. and Gallo, R.L. (2015) The role of the skin microbiome in atopic dermatitis. Curr. Allergy Asthma Rep. 15, 65

9. Kennedy, E.A. et al. (2017) Skin microbiome before development of atopic dermatitis: early colonization with commensal staphylococci at 2 months is associated with a lower risk of atopic dermatitis at 1 year. J. Allergy Clin. Immunol. 139, 166172

10. Nakatsuji, T. et al. (2017) Antimicrobials from human skin commensal bacteria protect against Staphylococcus aureus and are deficient in atopic dermatitis. Sci. Transl. Med. 9, eaah4680

11. Cogen, A.L. et al. (2010) Selective antimicrobial action is provided by phenol-soluble modulins derived from Staphylococcus epidermidis, a normal resident of the skin. J. Invest. Dermatol. $130,192-200$

12. Furue, M. et al. (2017) Atopic dermatitis: immune deviation, barrier dysfunction, IgE autoreactivity and new therapies. Allergol. Int. 66, 398-403

13. Salimi, M. et al. (2013) A role for IL-25 and IL-33-driven type-2 innate lymphoid cells in atopic dermatitis. J. Exp. Med. 210, 2939-2950

14. Cornelissen, C. et al. (2012) IL-31 regulates differentiation and filaggrin expression in human organotypic skin models. J. Allergy Clin. Immunol. 129, 426-433

15. Cevikbas, F. et al. (2014) A sensory neuron-expressed IL-31 receptor mediates T helper cell-dependent itch: Involvement of TRPV1 and TRPA1. J. Allergy Clin. Immunol. 133, 448-460

16. Czarnowicki, T. et al. (2015) Early pediatric atopic dermatitis shows only a cutaneous lymphocyte antigen (CLA)(+) TH2/TH1 cell imbalance, whereas adults acquire $\mathrm{CLA}(+) \mathrm{TH} 22 / \mathrm{TC} 22$ cell subsets. J. Allergy Clin. Immunol. 136, 941-951

17. Proksch, E. et al. (2008) The skin: an indispensable barrier. Exp. Dermatol. 17, 1063-1072

18. Chan, A. and Mauro, T. (2011) Acidification in the epidermis and the role of secretory phospholipases. Dermatoendocrinology 3 , 84-90

19. O'Regan, G.M. et al. (2008) Filaggrin in atopic dermatitis. J. Allergy Clin. Immunol. 122, 689-693

20. Kezic, S. et al. (2011) Levels of filaggrin degradation products are influenced by both filaggrin genotype and atopic dermatitis severity. Allergy 66, 934-940

21. Howell, M.D. et al. (2007) Cytokine modulation of atopic dermatitis filaggrin skin expression. J. Allergy Clin. Immunol. 120, 150155

22. Chieosilapatham, P. et al. (2017) Current insights into the role of human beta-defensins in atopic dermatitis. Clin. Exp. Immunol. $190,155-166$

23. Higaki, S. et al. (1999) Comparative study of staphylococci from the skin of atopic dermatitis patients and from healthy subjects. Int. J. Dermatol. 38, 265-269

24. Guzik, T.J. et al. (2005) Persistent skin colonization with Staphylococcus aureus in atopic dermatitis: relationship to clinical and immunological parameters. Clin. Exp. Allergy 35, 448-455

25. Park, H.Y. et al. (2013) Staphylococcus aureus colonization in acute and chronic skin lesions of patients with atopic dermatitis. Ann. Dermatol. 25, 410-416

26. Tauber, M. et al. (2016) Staphylococcus aureus density on lesional and nonlesional skin is strongly associated with disease severity in atopic dermatitis. J. Allergy Clin. Immunol. 137, 12721274

27. Totte, J.E. et al. (2016) Prevalence and odds of Staphylococcus aureus carriage in atopic dermatitis: a systematic review and meta-analysis. Br. J. Dermatol. 175, 687-695

28. Weidenmaier, C. et al. (2012) Staphylococcus aureus determinants for nasal colonization. Trends Microbiol. 20, 243-250

29. van Belkum, A. et al. (2009) Reclassification of Staphylococcus aureus nasal carriage types. J. Infect. Dis. 199, 1820-1826

30. Krismer, B. et al. (2017) The commensal lifestyle of Staphylococcus aureus and its interactions with the nasal microbiota. Nat. Rev. Microbiol. 15, 675-687

31. Feil, E.J. et al. (2003) How clonal is Staphylococcus aureus? J. Bacteriol. 185, 3307-3716

32. Monecke, S. et al. (2009) Molecular epidemiology of Staphylo coccus aureus in asymptomatic carriers. Eur. J. Clin. Microbiol. Infect. Dis. 28, 1159-1165

33. Melles, D.C. et al. (2004) Natural population dynamics and expansion of pathogenic clones of Staphylococcus aureus. J. Clin. Invest. 114, 1732-1740

34. Fleury, O.M. et al. (2017) Clumping factor B promotes adherence of Staphylococcus aureus to corneocytes in atopic dermatitis. Infect. Immun. 85, e00994-16 


\section{Trends in Microbiology}

35. Rojo, A. et al. (2014) Staphylococcus aureus genomic pattern and atopic dermatitis: may factors other than superantigens be involved? Eur. J. Clin. Microbiol. Infect. 33, 651-658

36. Yeung, M. et al. (2011) Identification of major clonal complexes and toxin producing strains among Staphylococcus aureus associated with atopic dermatitis. Microbes Infect. 13, 189-197

37. Clausen, M.L. et al. (2017) Staphylococcus aureus colonization in atopic eczema and its association with filaggrin gene mutations. Br. J. Dermatol. Published online September 24, 2017. http://dx.doi.org/10.1111/bjd.15470

38. Scharschmidt, T.C. et al. (2015) A wave of regulatory T cells into neonatal skin mediates tolerance to commensal microbes. Immunity 43, 1011-1021

39. Naik, S. et al. (2015) Commensal-dendritic-cell interaction specifies a unique protective skin immune signature. Nature 520, 104-108

40. Kong, H.H. et al. (2012) Temporal shifts in the skin microbiome associated with disease flares and treatment in children with atopic dermatitis. Genome Res. 22, 850-859

41. Byrd, A.L. et al. (2017) Staphylococcus aureus and Staphylococcus epidermidis strain diversity underlying pediatric atopic dermatitis. Sci. Transl. Med. 9, eaal4651

42. Zipperer, A. et al. (2016) Human commensals producing a novel antibiotic impair pathogen colonization. Nature 535, 511-516

43. Myles, I.A. et al. (2016) Transplantation of human skin microbiota in models of atopic dermatitis. JCl Insight 1, e86955

44. Ramsey, M.M. et al. (2016) Staphylococcus aureus shifts toward commensalism in response to Corynebacterium species. Front. Microbiol. 7, 1230

45. Shu, M. et al. (2013) Fermentation of Propionibacterium acnes, a commensal bacterium in the human skin microbiome, as skin probiotics against methicillin-resistant Staphylococcus aureus. PLoS One 8, e55380

46. Miajlovic, H. et al. (2010) Effect of filaggrin breakdown products on growth of and protein expression by Staphylococcus aureus. J. Allergy Clin. Immunol. 126, 1184-1190

47. Iwase, T. et al. (2010) Staphylococcus epidermidis Esp inhibits Staphylococcus aureus biofilm formation and nasal colonization. Nature 465, 346-349

48. Kobayashi, T. et al. (2015) Dysbiosis and Staphylococcus aureus colonization drives inflammation in atopic dermatitis. Immunity 42, 756-766

49. Cho, S.H. et al. (2001) Fibronectin and fibrinogen contribute to the enhanced binding of Staphylococcus aureus to atopic skin. J. Allergy Clin. Immunol. 108, 269-274

50. Foster, T.J. et al. (2014) Adhesion, invasion and evasion: the many functions of the surface proteins of Staphylococcus aureus. Nat. Rev. Microbiol. 12, 49-62

51. Mulcahy, M.E. et al. (2012) Nasal colonisation by Staphylococcus aureus depends upon clumping factor B binding to the squamous epithelial cell envelope protein loricrin. PLOS Pathog. 8, e1003092

52. Clarke, S.R. et al. (2007) The Staphylococcus aureus surface protein IsdA mediates resistance to innate defenses of human skin. Cell Host Microbe 1, 199-212

53. Clarke, S.R. et al. (2006) Identification of in vivo-expressed antigens of Staphylococcus aureus and their use in vaccinations for protection against nasal carriage. J. Infect. Dis. 193, 10981110

54. Corrigan, R.M. et al. (2009) Surface proteins that promote adherence of Staphylococcus aureus to human desquamated nasal epithelial cells. BMC Microbiol. 9, 22

55. Riethmuller, C. et al. (2015) Filaggrin breakdown products determine corneocyte conformation in patients with atopic dermatitis. J. Allergy Clin. Immunol. 136, 1573-1580

56. Spaulding, A.R. et al. (2013) Staphylococcal and streptococcal superantigen exotoxins, Clin. Microbiol. Rev. 26, 422-447
57. Berube, B.J. and Bubeck Wardenburg, J. (2013) Staphylococ cus aureus alpha-toxin: nearly a century of intrigue. Toxins (Basel) 5, 1140-1166

58. Sen, S. et al. (2016) Growth-environment dependent modulation of Staphylococcus aureus branched-chain to straight-chain fatty acid ratio and incorporation of unsaturated fatty acids. PLoS One 11, e0165300

59. Sun, Y. et al. (2012) Fatty acids regulate stress resistance and virulence factor production for Listeria monocytogenes. J. Bacteriol. 194, 5274-5284

60. Nguyen, M.T. et al. (2015) Skin-specific unsaturated fatty acids boost the Staphylococcus aureus innate immune response. Infect. Immun. 84, 205-215

61. Arad, G. et al. (2011) Binding of superantigen toxins into the CD28 homodimer interface is essential for induction of cytokine genes that mediate lethal shock. PLoS Biol. 9, e1001149

62. Herz, U. et al. (1998) A human-SCID mouse model for allergic immune response bacterial superantigen enhances skin inflammation and suppresses IgE production. J. Invest. Dermatol. $110,224-231$

63. Hu, D.L. and Nakane, A. (2014) Mechanisms of staphylococca enterotoxin-induced emesis. Eur. J. Pharmacol. 722, 95-107

64. Ono, H.K. et al. (2012) Submucosal mast cells in the gastrointestinal tract are a target of staphylococcal enterotoxin type A. FEMS Immunol. Med. Microbiol. 64, 392-402

65. Scheuber, P.H. et al. (1985) Skin reactivity of unsensitized monkeys upon challenge with staphylococcal enterotoxin B: a new approach for investigating the site of toxin action. Infect. Immun. 50, 869-876

66. Komisar, J. et al. (1992) Effects of staphylococcal enterotoxin B on rodent mast cells. Infect. Immun. 60, 2969-2975

67. Peschel, A. and Otto, M. (2013) Phenol-soluble modulins and staphylococcal infection. Nat. Rev. Microbiol. 11, 667-673

68. Nakamura, Y. et al. (2013) Staphylococcus delta-toxin induces allergic skin disease by activating mast cells. Nature 503, $397-$ 401

69. Nakagawa, S. et al. (2017) Staphylococcus aureus virulent PSM alpha peptides induce keratinocyte alarmin release to orchestrate IL-17-dependent skin inflammation. Cell Host Microbe 22, $667-677$

70. Syed, A.K. et al. (2015) Staphlyococcus aureus phenol-soluble modulins stimulate the release of proinflammatory cytokines from keratinocytes and are required for induction of skin inflam mation. Infect. Immun. 83, 3428-3437

71. Liu, H. et al. (2017) Staphylococcus aureus epicutaneous exposure drives skin inflammation via IL-36-mediated $T$ cell responses. Cell Host Microbe 22, 653-666

72. Breuer, K. et al. (2005) Alpha-toxin is produced by skin coloniz ing Staphylococcus aureus and induces a $T$ helper type 1 response in atopic dermatitis. Clin. Exp. Allergy 35, 1088-1095

73. Brauweiler, A.M. et al. (2014) Th2 cytokines increase Staphylococcus aureus alpha toxin-induced keratinocyte death through the signal transducer and activator of transcription 6 (STAT6). J. Invest. Dermatol. 134, 2114-2121

74. Brauweiler, A.M. et al. (2013) Filaggrin-dependent secretion of sphingomyelinase protects against staphylococcal alpha-toxininduced keratinocyte death. J. Allergy Clin. Immunol. 131, 421427

75. O'Halloran, D.P. et al. (2015) Protein A is released into the Staphylococcus aureus culture supernatant with an unprocessed sorting signal. Infect. Immun. 83, 1598-1609

76. Becker, S. et al. (2014) Release of protein A from the cell wall of Staphylococcus aureus. Proc. Natl. Acad. Sci. U. S. A. 111, 1574-1579

77. Gomez, M.l. et al. (2004) Staphylococcus aureus protein A induces airway epithelial inflammatory responses by activating TNFR1. Nat. Med. 10, 842-848 


\section{Trends in Microbiology}

78. Jun, S.H. et al. (2017) Staphylococcus aureus-derived membrane vesicles exacerbate skin inflammation in atopic dermatitis. Clin. Exp. Allergy 47, 85-96

79. Classen, A. et al. (2011) TNF receptor I on human keratinocytes is a binding partner for staphylococcal protein A resulting in the activation of NF kappa B, AP-1, and downstream gene transcription. Exp. Dermatol. 20, 48-52

80. Hashimoto, M. et al. (2006) Not lipoteichoic acid but lipoproteins appear to be the dominant immunobiologically active compounds in Staphylococcus aureus. J. Immunol. 177, 31623169

81. Nguyen, M.T. and Gotz, F. (2016) Lipoproteins of Gram-positive bacteria: key players in the immune response and virulence. Microbiol. Mol. Biol. Rev. 80, 891-903

82. Travers, J.B. et al. (2010) Infected atopic dermatitis lesions contain pharmacologic amounts of lipoteichoic acid. J. Allergy Clin. Immunol. 125, 146-152

83. Vu, A.T. et al. (2010) Staphylococcus aureus membrane and diacylated lipopeptide induce thymic stromal lymphopoietin in keratinocytes through the Toll-like receptor 2-Toll-like receptor 6 pathway. J. Allergy Clin. Immunol. 126, 985-993

84. Sonesson, A. et al. (2017) Identification of bacterial biofilm and the Staphylococcus aureus derived protease, staphopain, on the skin surface of patients with atopic dermatitis. Sci. Rep. 7 , 8689

85. Sieprawska-Lupa, M. et al. (2004) Degradation of human antimicrobial peptide LL-37 by Staphylococcus aureus-derived proteinases. Antimicrob. Agents Chemother. 48, 4673-4679

86. Nakatsuji, T. et al. (2016) Staphylococcus aureus exploits epidermal barrier defects in atopic dermatitis to trigger cytokine expression. J. Invest. Dermatol. 136, 2192-2200

87. Hirasawa, Y. et al. (2010) Staphylococcus aureus extracellular protease causes epidermal barrier dysfunction. J. Invest. Dermatol. 130, 614-617

88. Williams, M.R. et al. (2017) Staphylococcus aureus induces increased serine protease activity in keratinocytes. J. Invest. Dermatol. 137, 377-384

89. Stentzel, S. et al. (2017) Staphylococcal serine protease-like proteins are pacemakers of allergic airway reactions to Staphylococcus aureus. J. Allergy Clin. Immunol. 139, 492-500
90. Carr, W.W. (2013) Topical calcineurin inhibitors for atopic dermatitis: review and treatment recommendations. Paediatr. Drugs 15, 303-310

91. Flohr, C. and Irvine, A.D. (2013) Systemic therapies for severe atopic dermatitis in children and adults. J. Allergy Clin. Immunol. 132, 774-774

92. Brunner, P.M. et al. (2017) The immunology of atopic dermatitis and its reversibility with broad-spectrum and targeted therapies. J. Allergy Clin. Immunol. 139, S65-S76

93. Huang, J.T. et al. (2009) Treatment of Staphylococcus aureus colonization in atopic dermatitis decreases disease severity. Pediatrics 123, e808-14

94. Francis, N.A. et al. (2017) Oral and topical antibiotics for clinically infected eczema in children: a pragmatic randomized controlled trial in ambulatory care. Ann. Fam. Med. 15, 124-130

95. Pastagia, M. et al. (2013) Lysins: the arrival of pathogen-directed anti-infectives. J. Med. Microbiol. 62, 1506-1516

96. Herpers, B.L. and Leeson, N. (2015) Endolysins: redefining antibacterial therapy. Future Microbiol. 10, 309-311

97. Totte, J.E.E. et al. (2017) Successful treatment of chronic Staphylococcus aureus-related dermatoses with the topical endolysin Staphefekt SA.100: a report of 3 cases. Case Rep. Dermatol. 9 , 19-25

98. Formosa-Dague, C. et al. (2016) Forces between Staphylococcus aureus and human skin. Nanoscale Horizons 1, 298-303

99. Bunikowski, R. et al. (2000) Evidence for a disease-promoting effect of Staphylococcus aureus-derived exotoxins in atopic dermatitis. J. Allergy Clin. Immunol. 105, 814-819

100. Leung, D.Y. et al. (1993) Presence of lgE antibodies to staphylococcal exotoxins on the skin of patients with atopic dermatitis. Evidence for a new group of allergens. J. Clin. Invest. 92, 1374 1380

101. Bunikowski, R. et al. (1999) Prevalence and role of serum lgE antibodies to the Staphylococcus aureus-derived superantigen SEA and SEB in children with atopic dermatitis. J. Allergy Clin Immunol. 103, 119-124

102. Skov L etal. (2000) Application of staphylococcal enterotoxin B on normal and atopic skin induces up-regulation of $\mathrm{T}$ cells by a superantigen-mediated mechanism. J. Allergy Clin. Immunol. 105, 820-826 